\title{
IMPLICATIONS OF A STATE-SPACE APPROACH FOR THE ECONOMIC OPTIMAL DESIGN OF A NGCC POWER PLANT
}

\author{
E. GODOY ${ }^{1}$, S. J. BENZ ${ }^{1}$ and N. J. SCENNA ${ }^{1,2}$ \\ ${ }^{1}$ Centro de Aplicaciones Informáticas y Modelado en Ingeniería, Facultad Regional Rosario, \\ Universidad Tecnológica Nacional (CAIMI, FRRO, UTN) \\ ${ }^{2}$ Consejo Nacional de Investigaciones Científicas y Técnicas (CONICET) \\ E-mail contact: caimi@frro.utn.edu.ar
}

\begin{abstract}
A detailed state-space approach is here embedded in the optimization model for the economic design of a new natural gas combined cycle (NGCC) power plant. This proposal comprehensively quantifies the impact of different adopted values for two critical parameters: the number of simultaneously and independently failed components, and the number of simultaneous events.

The former depicts the maximum number of components (gas turbines, steam turbine, HRSGs, auxiliary services, etc.) that seem feasible to fail because of independent causes at the same time. The latter describes the maximum number of transitions between operating conditions, which is directly linked to the amount of available maintenance resources (personnel, parts, etc).

Implications of a wide array of values for these two parameters over the economic optimal solutions are thoroughly discussed, where it is observed that more accurate and detailed estimations of the expected economic performance for the project are attained.
\end{abstract}

\section{INTRODUCTION}

In order to consider the effects of availability and maintenance in the plant economics, Goel et al (2002, 2003) indicated that revenues and operating costs must be affected by the system inherent availability. Aguilar et al (2008) incorporated reliability and availability into the design (configuration and redundancy allocation) and operation (maintenance schedule) of flexible utility plants; and observed that two different tradeoffs may arise: capital investment versus contractual penalties for not fulfilling the power demand (which can be computed as profit losses during the plant down time); and capital investment versus costs originated by different failure scenarios (while evaluating if the plant is able to cope with the demand).

Applying the state-space method consists of three steps, as identified by El-Nashar (2008): first, identification of functional and failure modes of the system by making an inventory of all possible states; second, establishment of rules for transition between states and formulation of the transition rate matrix; and third, evaluation of expected values of the interest variables, by using the states probabilities as weights. 


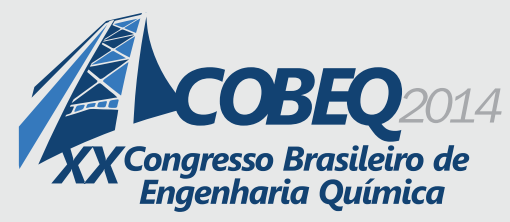

19 a 22 de outubro de 2014

Florianópolis/SC

Terrazas-Morenoo et al. (2010) proved that it is possible to optimize over the state-space in the last level of the tree, even if the optimal flowsheet contains less units than those present in the last level; as the states in the last level of the tree that are derived from the same node are identical in terms of feasibility/infeasibility, and also, the sum of the probabilities of the states in the last level of the tree is equal to the probability of the state from which they are derived. They also observed that there are two ways for reducing the number of possible states: first, the failures with the same related rate reduction that occur in the same plant can be aggregated as one equivalent failure; and in second place, only the most probable states can be considered (as for example, those ones that cover $99 \%$ of the long term operating horizon).

In this work, a state-space approach is used to account for the availability of the generation plant, thus facilitating a complete overview of the system operating condition across the entire time horizon, which also enables more accurately computing the optimal economic performance of the project. The increased accuracy is achieved by weighting the economic performance indicators across every feasible operating scenario that the plant has to deal with, while considering their functionality on the availability related variables.

The resultant MINLP model provides economic optimal solutions for the design and operation of the NGCC power plant, as it has embedded every step required for the comprehensive application of the state-space approach for determining the probability of occurrence of each functional status, while implementing efficient strategies for identifying the operating scenarios which exert the larger impact on the project economics.

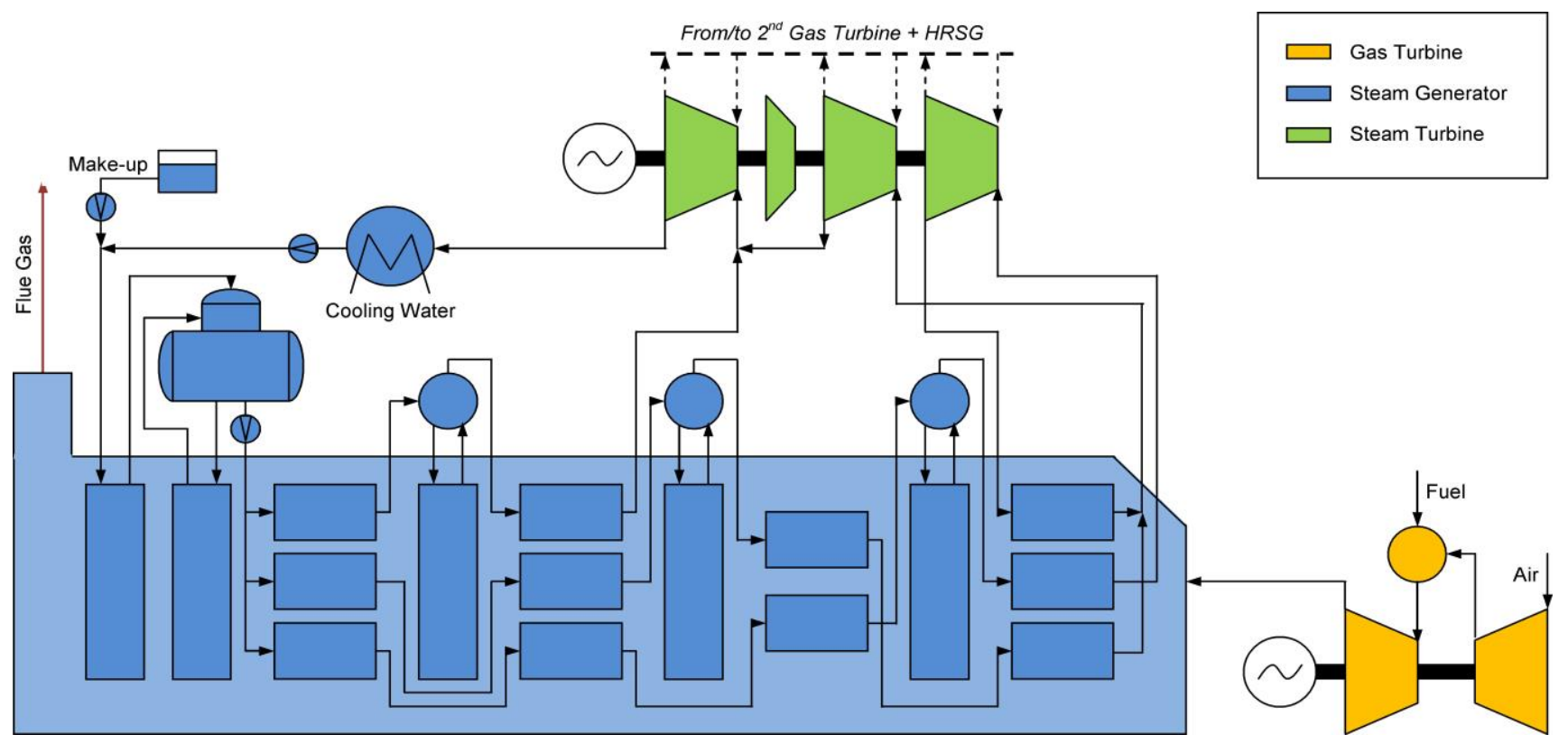

Figure 1 - Flow diagram for the NGCC power plant. 


\section{OPTIMIZATION PROBLEM}

\subsection{Mathematical Optimization Formulation}

The flow diagram for the NGCC power plant is presented in Figure 1. A 2 GTs + 1 ST multishaft power plant is selected as the generation driver (note that the second gas turbine and its associated steam generator are not presented in this figure).

The mathematical formulation for the economic optimization of the NGCC power plant is presented at Figure 2. In this optimization problem, the cost of the generated electricity COE is selected as objective function $f$; which implies simultaneously minimizing the total expenditures of the project and maximizing the net energy output of the plant. Here, $x$ are the sets of design and operating variables and $\underline{y}$ are the sets of integer variables; while $\underline{h}$ and $\underline{g}$ refer to the equality and inequality constraints which configure the mathematical model of the whole project. A complete description of the NGCC power plant model is presented by Godoy et al. $(2010,2011)$.

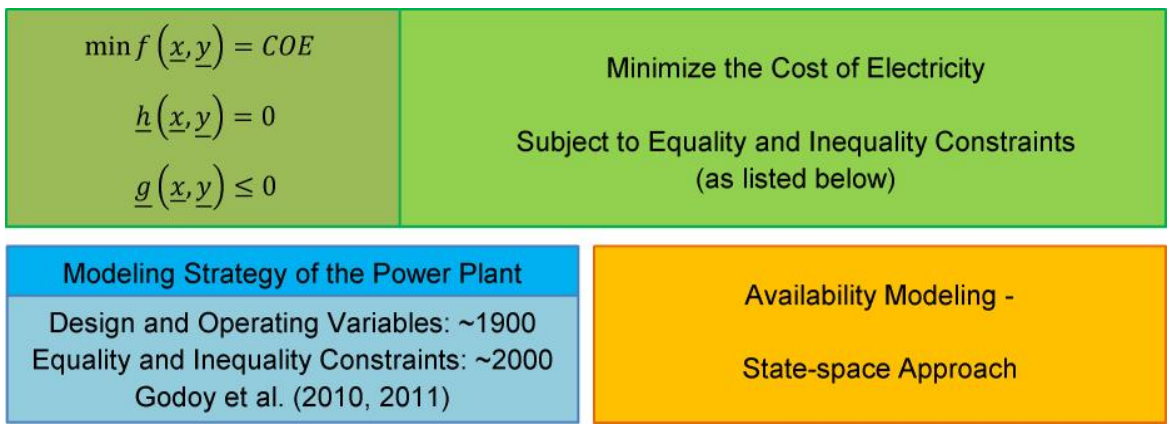

Figure 2 - Economic optimization problem.

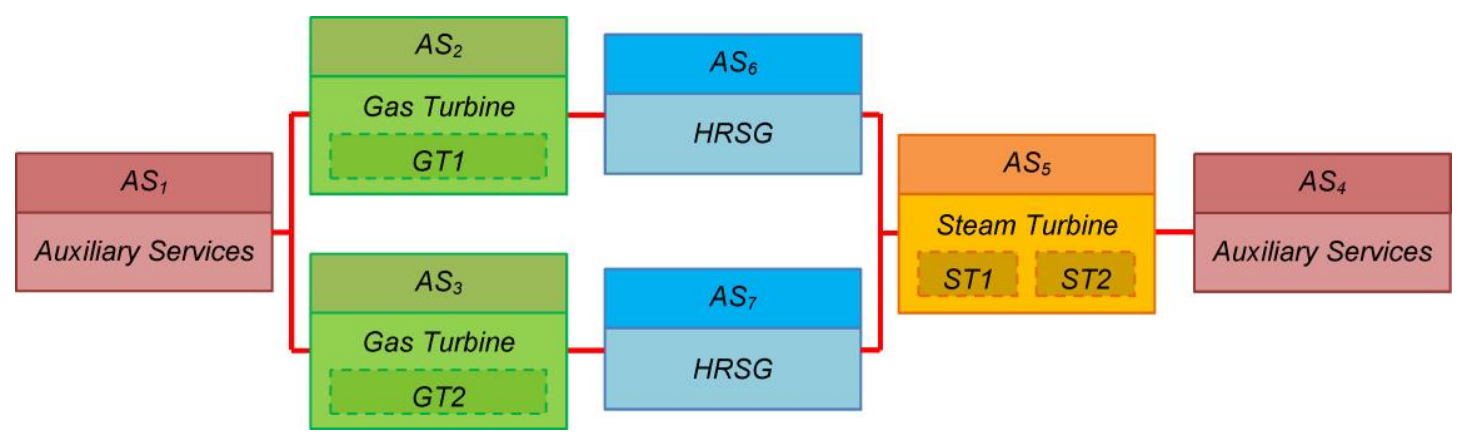

Figure 3 - Reliability block diagram for the NGCC power plant. 


\subsection{State-Space Approach}

The state-space method (i.e. a Markov-type approach) is used to evaluate the probability of the system being at each functional mode. These probabilities can be used for computing the operability indices of the system, as well as evaluating its technical and economic performance indicators.

The reliability block diagram associated to the power plant is introduced in Figure 3.

Altogether, the overall functional status of the power plant can be determined as the conjunction of the operating condition of every component, as described in Table 1 regarding the delivered energy (and eventually, the consumed resources).

Table 1 - Description of functional statuses for the NGCC power plant.

\begin{tabular}{|c|c|}
\hline $\begin{array}{l}\text { Functional } \\
\text { Status }\end{array}$ & Description \\
\hline P1 & The power plant operates at full capacity \\
\hline $\mathrm{P} 2$ & $\begin{array}{l}\text { Both gas turbines operate at full capacity, the steam turbine operates at } \\
\text { half capacity }\end{array}$ \\
\hline P3 & Both gas turbines operate at open loop \\
\hline P4 & $\begin{array}{l}\text { Only one gas turbine operates at full capacity, the other gas turbine is } \\
\text { down, the steam turbine operates at half capacity }\end{array}$ \\
\hline P5 & $\begin{array}{l}\text { Only one gas turbine operates at open loop, the other gas turbine and } \\
\text { the steam turbine are down }\end{array}$ \\
\hline P6 & The power plant is down \\
\hline
\end{tabular}

\subsection{Number of functional modes and their interrelations}

The number of functional modes of the system and their interrelations depend upon the values of two parameters: the number of simultaneously and independently failed components $N_{S I F C}$ and the number of simultaneous events $N_{S E}$, where it is observed that:

- Each possible functional mode is defined by the set of binary variables associated to every component, where an operating status is represented by a 1 , while a 0 depicts a failed status

- The maximum number of zeroes at each feasible functional mode is given by $N_{S I F C}$. Only a state-space where $N_{S I F C}$ equals the number of components in the system will include a mode where every component is failed

- Transitions between modes occur as the operating condition of one or more components change 
from operative to failed (from 1 to 0 ), or vice versa; where the maximum number of allowable simultaneous events is given by the value fixed by the designer for $N_{S E}$. For example, if $N_{S E}$ equals 2, the state-space will include transitions between feasible modes which imply simultaneously changing the operating status of two components (i.e. two failures, two repair actions, or a failure and a repair action)

For different values of the number of simultaneously and independently failed components $N_{S I F C}$ and the number of simultaneous events $N_{S E}$, Figure 4 presents the number of feasible functional modes and the number of interconnections between them, respectively. It is here observed that:

- The number of feasible functional modes depends on the value adopted for $N_{S I F C}$, while is independent of $N_{S E}$

- The number of interconnections between modes increases as $N_{S I F C}$ and $N_{S E}$ do

- While $N_{S I F C}$ is lower than the number of components, the state-space method is not able to represent every possible functional mode

\begin{tabular}{|c|c|c|c|c|}
\cline { 2 - 5 } \multicolumn{1}{c|}{} & \multicolumn{4}{c|}{$N_{S E}$} \\
\hline$N_{\text {SIFC }}$ & 1 & 2 & 3 & 4 \\
\hline 1 & 8 & 8 & 8 & 8 \\
\hline 2 & 29 & 29 & 29 & 29 \\
\hline 3 & 64 & 64 & 64 & 64 \\
\hline 4 & 99 & 99 & 99 & 99 \\
\hline 5 & 120 & 120 & 120 & 120 \\
\hline 6 & 127 & 127 & 127 & 127 \\
\hline 7 & 128 & 128 & 128 & 128 \\
\hline
\end{tabular}

a. Number of feasible functional modes.

\begin{tabular}{|r|r|r|r|r|}
\cline { 2 - 5 } \multicolumn{1}{c|}{} & \multicolumn{4}{|c|}{$N_{\text {SE }}$} \\
\hline$N_{\text {SIFC }}$ & 1 & 2 & 3 & 4 \\
\hline 1 & 7 & 28 & 28 & 28 \\
\hline 2 & 49 & 196 & 301 & 406 \\
\hline 3 & 154 & 616 & 1176 & 1736 \\
\hline 4 & 294 & 1176 & 2506 & 3836 \\
\hline 5 & 399 & 1596 & 3556 & 5516 \\
\hline 6 & 441 & 1764 & 3969 & 6174 \\
\hline 7 & 448 & 1792 & 4032 & 6272 \\
\hline
\end{tabular}

b. Number of interconnections.

Figure 4 - Number of feasible functional modes and their interconnections.

\section{OPTIMAL DESIGNS: RESULTS AND DISCUSSION}

Optimal designs for the NGCC power plant are here obtained by solving the economic optimization formulation previously detailed, in order to analyze the influence of modifications on the values adopted for the availability related parameters over the optimal economics of the generation project. Then, the number of simultaneously and independently failed components $N_{S I F C}$ is varied from 1 to 7 , while the number of simultaneous events $N_{S E}$ is varied from 1 to 4 .

Figure 5 presents the optimal economic indicators of the project for each pair of values of $N_{S I F C}$ and $N_{S E}$. The total annual cost decreases as the value of $N_{S I F C}$ increases, for a given value of $N_{S E}$, as shown in Figure 5.a. The main cost components exhibit similar trends, as illustrated for the fuel consumption in Figure 5.b. 


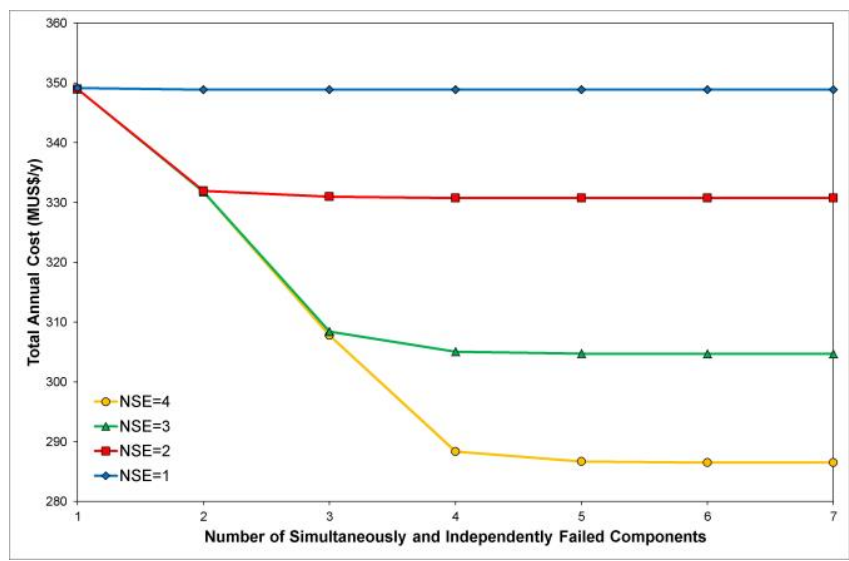

a. Total annual cost.

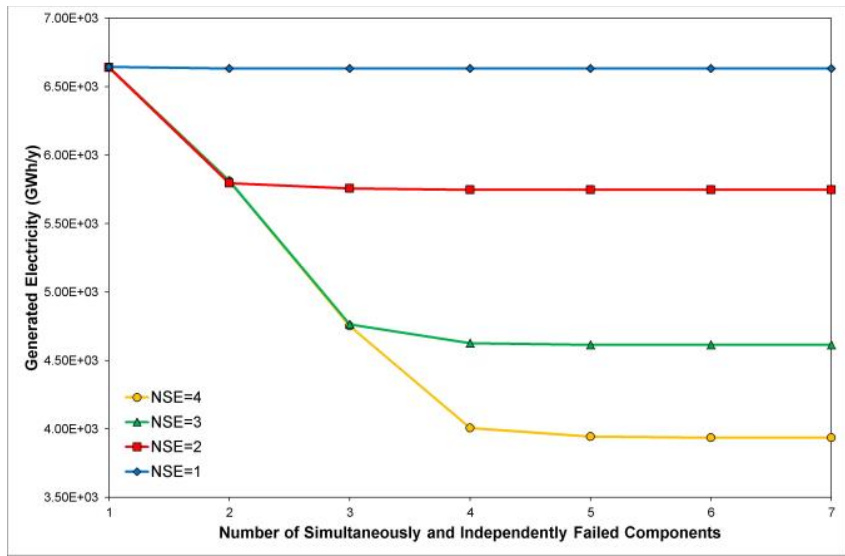

c. Generated electricity.

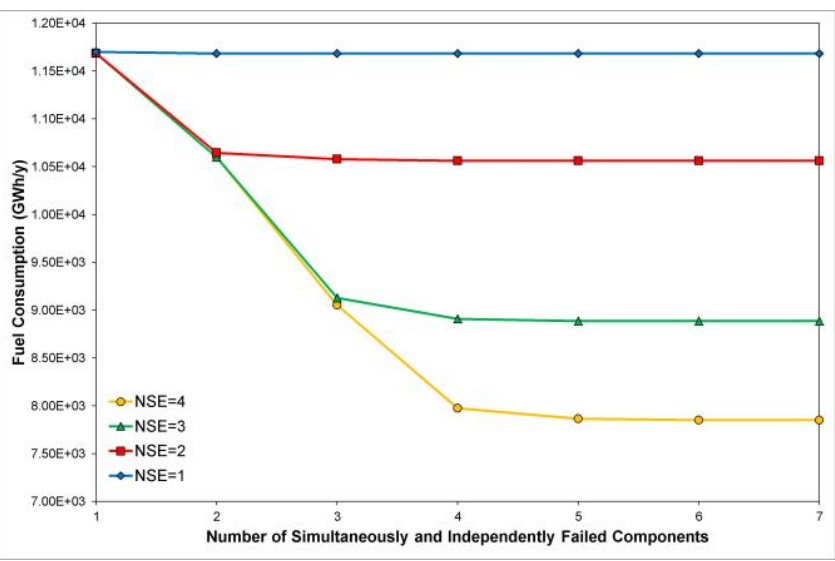

b. Fuel consumption.

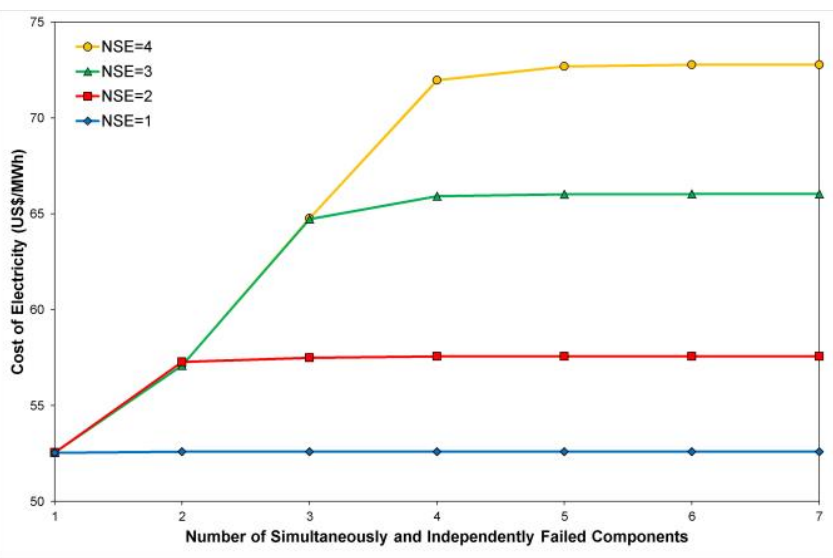

d. Cost of electricity.

Figure 5 - Economic indicators for different $N_{S I F C}$ and $N_{S E}$ values.

It is also observed in Figure 5.c that the total delivered energy becomes smaller, as the values of $N_{S I F C}$ and $N_{S E}$ increase. Since the total annual cost decreases at a lower rate than the delivered energy's, it is observed in Figure 5.d that the cost of the generated electricity increases as the value of $N_{S I F C}$ does, for a given value of $N_{S E}$.

The observed trends regarding the evolution of the objective function can then be explained when considering the probability of occurrence of each feasible functional mode, since operating at a derated condition is more economically inefficient, and consequently implies a larger average (or weighted) cost per unit of delivered energy. 


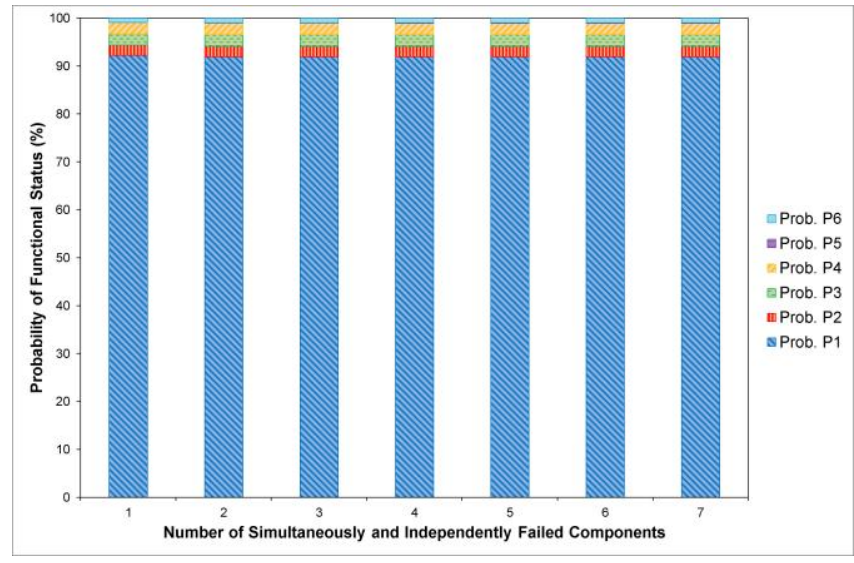

a. Total annual cost.

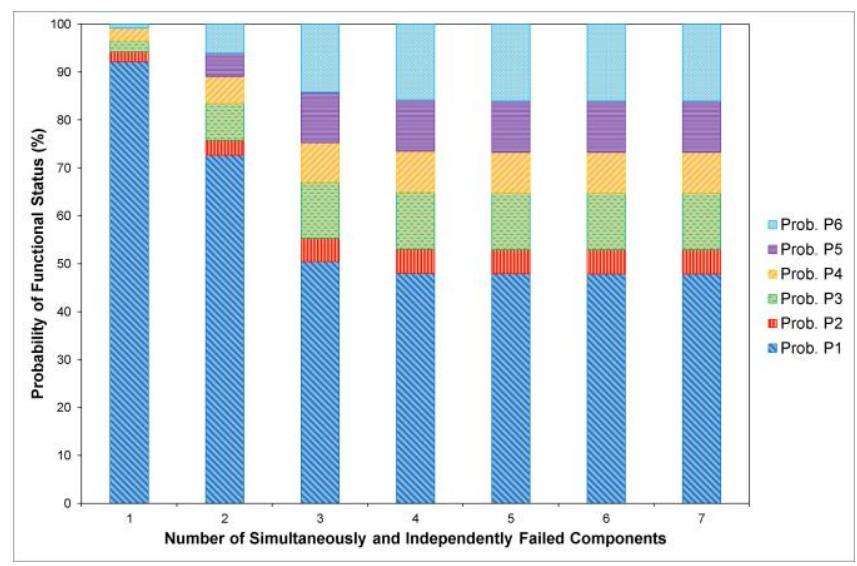

c. Generated electricity.

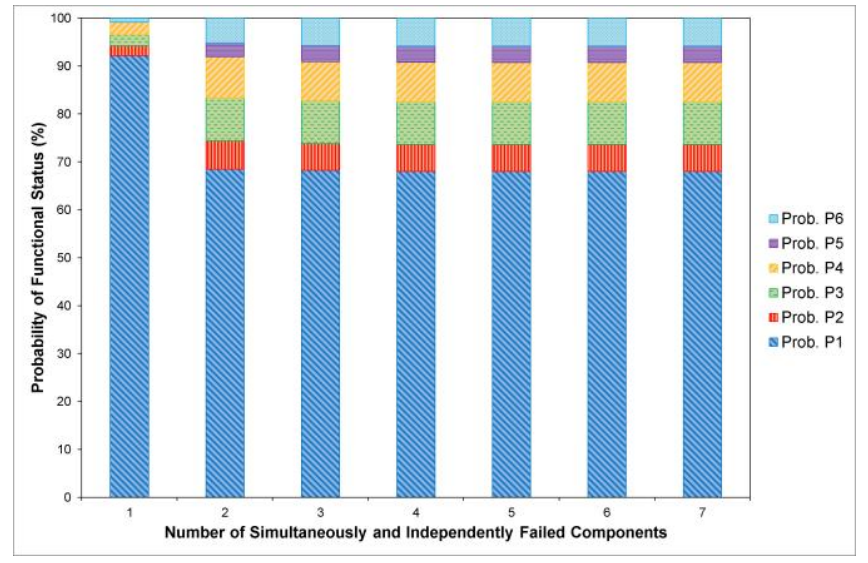

b. Fuel consumption.

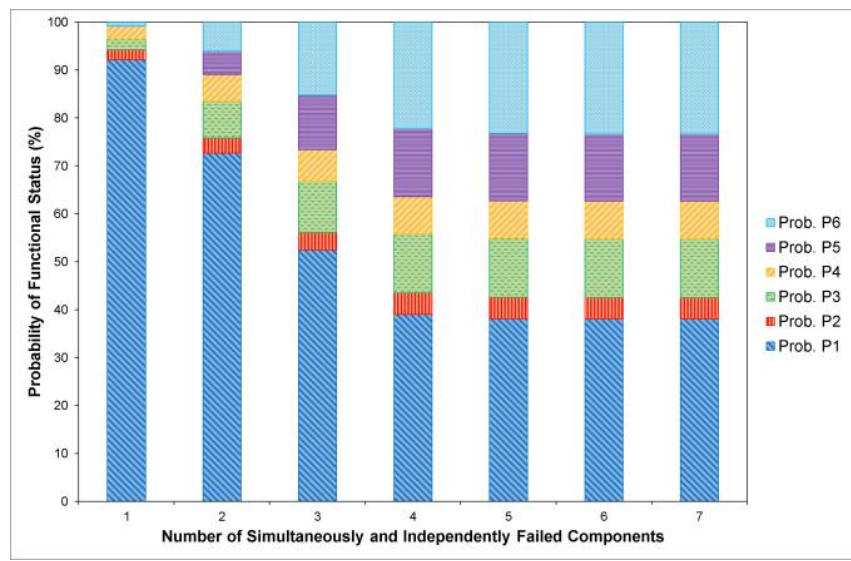

d. Cost of electricity.

Figure 6 - Probability of functional statuses for different $N_{S I F C}$ and $N_{S E}$ values.

The probabilities of occurrence of each feasible functional mode are introduced at Figure 6. It is here observed that:

- For $N_{S E}$ equal to 1 , the probabilities remain almost invariant for the whole range of values of $N_{S I F C}$

- As $N_{S E}$ adopts a value equal or greater than 2, the functional statuses which represent derated operating conditions become increasingly more common, while the probability of operating at full capacity falls exponentially 


\section{CONCLUSIONS}

A comprehensive strategy has been here discussed in order to successfully achieve the economic optimization of a NGCC power plant, while considering the availability of the system through its wide array of feasible operating statuses by means of a state-space approach.

It is here found that values of parameters $N_{S I F C}$ and $N_{S E}$ are critical for the successful evaluation of the optimal economic performance of the generation plant. It is then observed that the value for both $N_{S I F C}$ and $N_{S E}$ should be carefully selected in order to better depict the actual characteristics of NGCC power plants across the full span of functional statuses comprised in the time horizon.

Even though, restraint should be exercised since the size of the resultant optimization mathematical problem becomes larger as the value of $N_{S I F C}$ does, also requiring extra computational resources in order to promptly achieve convergence.

\section{ACKNOWLEDGEMENTS}

The authors gratefully acknowledge the financial support of the Agencia Nacional de Promoción Científica y Tecnológica (ANPCyT), the Universidad Tecnológica Nacional (UTN) and the Consejo Nacional de Investigaciones Científicas y Técnicas (CONICET).

\section{REFERENCES}

AGUILAR, O., KIM, J., PERRY, S., SMITH, R., 2008. Availability and reliability considerations in the design and optimization of flexible utility systems. Chem. Eng. Sci. 63 (14), 3569-3584.

EL-NASHAR, A., 2008. Optimal design of a cogeneration plant for power and desalination taking equipment reliability into consideration. Desalination 229 (1-3), 21-32.

GODOY, E., BENZ, S., SCENNA, N., 2011. A strategy for the economic optimization of combined cycle gas turbine power plants by taking advantage of useful thermodynamic relationships. Appl. Therm. Eng. 31, 852-871.

GODOY, E., SCENNA, N., BENZ, S., 2010. Families of optimal thermodynamic solutions for combined cycle gas turbine (CCGT) power plants. Appl. Therm. Eng. 30 (6-7), 569-576.

GOEL, H., GRIEVINK, J., HERDER, P.,WEIJNEN, M., 2002. Integrating reliability optimization into chemical process synthesis. Reliab. Eng. Syst. Safe. 78 (3), 247-258.

GOEL, H., GRIEVINK, J., HERDER, P., WEIJNEN, M., 2003. Optimal reliability design of process systems at the conceptual stage of design. In: Annual Reliability and Maintainability Symposium, IEEE, pp. 40-45.

TERRAZAS-MORENO, S., GROSSMANN, I., WASSICK, J., BURY, S., 2010. Optimal design of reliable integrated chemical production sites. Comp. Chem. Eng. 34 (12), 1919-1936. 